OPEN ACCESS

Edited by:

Bart Ellenbroek

Victoria University of Wellington,

New Zealand

Reviewed by:

Tianmei Si,

Peking University Sixth Hospital, China

Jari Lahti,

University of Helsinki, Finland

*Correspondence:

Sandra Carvalho

sandrarc@psi.uminho.pt

Specialty section:

This article was submitted to

Molecular Psychiatry,

a section of the journal

Frontiers in Psychiatry

Received: 15 June 2020

Accepted: 01 October 2020

Published: 03 November 2020

Citation:

Carvalho S, Gonçalves ÓF, Brunoni AR, Fernandes-Gonçalves A,

Fregni F and Leite J (2020)

Transcranial Direct Current Stimulation

as an Add-on Treatment to

Cognitive-Behavior Therapy in First

Episode Drug-Naive Major Depression

Patients: The ESAP Study Protocol.

Front. Psychiatry 11:563058.

doi: 10.3389/fpsyt.2020.563058

\section{Transcranial Direct Current} Stimulation as an Add-on Treatment to Cognitive-Behavior Therapy in First Episode Drug-Naïve Major Depression Patients: The ESAP Study Protocol

\author{
Sandra Carvalho ${ }^{1,2 *}$, Óscar F. Gonçalves ${ }^{1,2,3}$, André R. Brunoni ${ }^{4}$, \\ Ana Fernandes-Gonçalves ${ }^{5}$, Felipe Fregni ${ }^{2}$ and Jorge Leite ${ }^{2,6}$
}

${ }^{1}$ Psychological Neuroscience Laboratory, CIPsi, School of Psychology, University of Minho, Braga, Portugal, ${ }^{2}$ Department of Physical Medicine and Rehabilitation, Spaulding Neuromodulation Center, Spaulding Rehabilitation Hospital, Massachusetts General Hospital and Harvard Medical School, Boston, MA, United States, ${ }^{3}$ Proaction Laboratory, Faculty of Psychology and Educational Sciences, University of Coimbra, Coimbra, Portugal, ${ }^{4}$ Department and Institute of Psychiatry, Service of Interdisciplinary Neuromodulation, Laboratory of Neurosciences (LIM-27), Interdisciplinary Center for Applied Neuromodulation University Hospital, University of São Paulo, São Paulo, Brazil, ${ }^{5}$ Department of Psychiatry, CUF Porto Hospital, Porto, Portugal, ${ }^{6}$ Univ Portucalense, Portucalense Institute for Human Development-INPP, Porto, Portugal

Background: Major Depressive Disorder (MDD) affects more than 264 million people worldwide. Current treatments include the use of psychotherapy and/or drugs, however $\sim 30 \%$ of patients either do not respond to these treatments, or do not tolerate the side effects associated to the use of pharmacological interventions. Thus, it is important to study non-pharmacological interventions targeting mechanisms not directly involved with the regulation of neurotransmitters. Several studies demonstrated that transcranial Direct Current Stimulation (tDCS) can be effective for symptoms relief in MDD. However, tDCS seems to have a better effect when used as an add-on treatment to other interventions.

Methods/Design: This is a study protocol for a parallel, randomized, triple-blind, sham-controlled clinical trial in which a total of 90 drug-naïve, first-episode MDD patients (45 per arm) will be randomized to one of two groups to receive a 6-weeks of CBT combined with either active or sham tDCS to the dorsolateral prefrontal cortex (DLPFC). The primary outcome will depressive symptoms improvement as assessed by the Montgomery-Asberg Depression Rating Scale (MADRS) at 6-weeks. The secondary aim is to test whether CBT combined with tDCS can engage the proposed mechanistic target of restoring the prefrontal imbalance and connectivity through the bilateral modulation of the DLPFC, as assessed by changes over resting-state and emotional task eliciting EEG.

Discussion: This study evaluates the synergetic clinical effects of CBT and tDCS in the first episode, drug-naiive, patients with MDD. First episode MDD patients provide an interesting opportunity, as their brains were not changed by the pharmacological treatments, by the time course, or by the recurrence of MDD episodes (and other comorbidities). 
Trial Registration: This study is registered with the United States National Library of Medicine Clinical Trials Registry (NCT03548545). Registered June 7, 2018, clinicaltrials.gov/ct2/show/NCT03548545. Protocol Version 1.

Keywords: MDD (Major Depressive Disorder), study protocol, drug-naïve, tDCS (transcranial direct current stimulation), CBT (cognitive-behavioral therapy)

\section{BACKGROUND}

Major Depressive Disorder(MDD) is widely recognized as a staggering global healthcare challenge, as well as a potentially lethal illness (1). The worldwide prevalence of Depression is about $3.4 \%$ [2-6\%], and mild forms of depression are the most prevalent $-13 \%$, as compared to $4 \%$ for moderate forms and 5.1 $\%$ for severe forms of depression (2). The prevalence in females is about $4.1 \%$ and about $2.7 \%$ in males (2). Overall, MDD is thought to affect 264 million people worldwide, thus ranking second in the most common causes of disability with prospects of becoming the first by 2040 (3).

The current standard care for MDD involves the use of psychotherapy, antidepressant medication, or a combination of both. Despite the costs involved in these interventions, the efficacy of such treatments may have been overestimated, with recent data suggesting that remission rates can be as low as $23 \%$ depending on the self-report scale used (4). Furthermore, 30\% of patients suffering from MDD still exhibit depressive symptoms despite the appropriate psychological and pharmacological treatments (5). In order to overcome this, several treatments are frequently combined, usually by the use of drug augmentation and/or combination of different drugs, which often increases the risk of adverse effects (6). Thus, the development of effective treatment alternatives for MDD, which includes non-pharmacological interventions targeting mechanisms not directly involved with the regulation of neurotransmitters, is an urgent research priority. However, in order to do so, it is important to understand the underlying neural mechanisms involved in MDD.

Evidence coming from several electroencephalography (EEG) (7-9), neuroimaging $(10,11)$, and neuromodulation (12-15) studies showed that the dorsolateral prefrontal cortex (DLPFC) is as an important area of dysfunction in depression, mainly due its left hypo and right hyper-functioning. This interhemispheric imbalance over the DLPFC has been shown to be an indicator of lifetime MDD, or in conjunction with depressive self-schema (i.e., an interconnected negative internal representation of the self that has been associated to the onset and maintenance of depressive state $(16,17)$ to be a predictor of a first prospective MDD episode (18). Nevertheless, some studies failed to show the link between decreased left frontal activation and depression (19).

Transcranial direct current stimulation (tDCS) is a noninvasive method of brain stimulation that is capable of depolarizing or hyperpolarizing the neural membrane and as such, it has been used in people suffering from depression, by placing the anode (excitatory) over the left and the cathode (inhibitory) over the right DLPFC, or by placing the anode over the left DLPFC and the cathode over the right supra-orbital region.

For instance, in one study, $64 \mathrm{MDD}$ patients were randomized to 15 sessions of $2 \mathrm{~mA}$ tDCS over 3 weeks, and tDCS was shown to be able to decreased MDD symptoms (20). However, in another study, not only active tDCS was superior to sham but also the combination of tDCS with sertraline was significantly more effective in reducing depressive symptoms than either treatment alone (21). Although tDCS per se showed promising results in treating MDD, the previous trial highlights that the effects of tDCS can be enhanced by combining it with other interventions. Overall, tDCS seems to decrease MDD symptoms by a pooled effect size of 0.36 (22). Moreover, according to a recent individual patient data meta-analysis, tDCS seems to be less effective in high-resistant patients, suggesting that tDCS may be a promising add-on therapy to therapies such as the cognitive behavioral therapy (CBT) (23) or cognitive control therapy $(24,25)$.

CBT is an empirically validated therapy for the treatment of MDD. Several studies have demonstrated the efficacy of CTB alone $(26,27)$ or as adjuvant to medication $(28-30)$ in acute depression. Another advantage of CBT over anti-depressant drugs is its long-term effects, namely protecting against relapses and recurrences after active treatments has ended $(27,31,32)$. Additionally, CBT is a well-established therapy that can restore or normalize abnormal brain activity, namely prefrontal alpha activity (33). Namely, an increase in left frontal brain activity after CBT in individuals with anxiety and depression.

It is important to highlight that MDD seems to induce profound changes in the brain, namely structural alterations in fronto-cingulate-striatal circuits (34-36). However, and somewhat surprisingly, first episode MDD patients have not been extensively studied with tDCS $(37,38)$. These first episode MDD patients provide an interesting opportunity, as their brains were not changed by the pharmacological treatments, by the time course, or by the recurrence of MDD episodes (and other comorbidities).

\section{PURPOSE, PRIMARY AND SECONDARY OUTCOMES}

Therefore, we propose to study the clinical and mechanistic effects of the combination of two well-studied interventionsCBT and tDCS - for the treatment of MDD in drug naïve first episode patients. The primary outcome will be the clinical effects (severity of depression/mood amelioration), as measured by the Montgomery-Asberg Depression Rating Scale (MADRS). Secondary outcomes will be resting state and emotional task eliciting EEG, which will be useful simultaneously to understand 
the neural effects of the intervention, as well as potential response predictors for future trials.

\section{RESEARCH QUESTION, AIMS, HYPOTHESIS}

By combining two interventions that showed promising results in MDD (CBT and tDCS), we aim to investigate the clinical and underlying neurophysiological effects in first-episode drug naive MDD patients. The main research question underlying this proposal is whether tDCS as add-on therapy to CBT in drug naïve, first-episode MDD patients could produce greater significant clinical improvements, as measured by the MADRS, when compared to CBT alone. We hypothesize that the combination of these therapies will produce a synergetic effect in the brain, potentiating the effects of CBT and decreasing the depressive symptomatology as assessed by the MADRS.

A secondary aim will be to perform the dose calculation (number of sessions) required to induce a clinically significant effect $(50 \%$ decrease in the MADRS score). The underlying hypothesis is that the combination of tDCS with CBT will require a lesser number of sessions in order to elicit this clinical meaningful effect.

The second major scientific question is whether these clinical improvements will be correlated with the rebalancing of the inter-hemispheric asymmetry of EEG alpha activity toward the left hemisphere, as assessed by resting-state and emotional task eliciting-EEG. Here, the hypothesis is that both interventions (combined and alone) will reduce the inter-hemispheric alpha imbalance, as indexed by EEG power; however, that reduction will be more pronounced in the group that received the add-on intervention, as compared to CBT alone.

\section{METHODS}

\section{Trial Design, Setting and Registration}

This is a parallel, randomized, triple-blind, sham controlled clinical protocol in which a total of 90 drug-naïve, first-episode MDD outpatients (45 per arm) will be randomized to one of two groups: active bilateral tDCS over the DLPFC combined with CBT or sham tDCS combined with CBT (Figure 1).

Patients will complete a 6-weeks treatment that involves 18 tDCS sessions (active or sham) and 12 CBT sessions. For the first 2 weeks of intervention, they will receive 10 daily sessions of tDCS (from Monday to Friday) and 4 sessions of CBT (combined at same time with tDCS for the first $30 \mathrm{~min}$, on Monday and on Friday). They will then receive two booster sessions of tDCS combined with CBT will be on weeks 3, 4, 5, and 6 (Figure 2B).

This study will then be organized in 3 distinct phases. Phase 1: Recruitment and baseline assessments; Phase 2: Intervention; Phase 3: Post-treatment assessment and 6-month follow-up (Figure 1). Once eligibility is determined and consent provided, patients will be randomly assigned to one of the two groups in a 1:1 allocation ratio, by the means of a list generated by an automatic web-based randomization program.

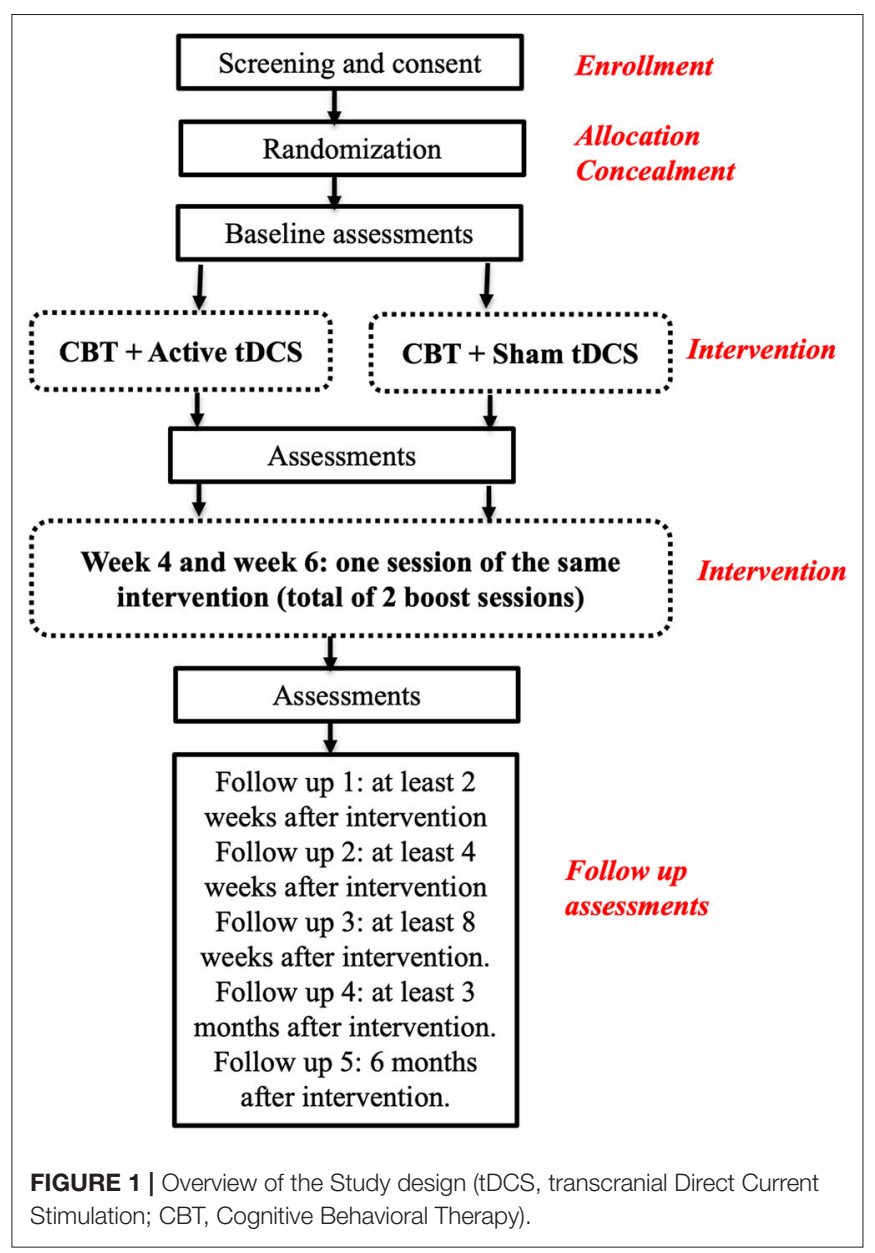

After an initial baseline assessment (visit 1) (for more details see task 1), patients will receive 10 consecutive sessions (in two weeks) of active or sham tDCS $(2 \mathrm{~mA}, 30 \mathrm{~min})$, and 6 sessions of CBT every other day (3 CBT sessions per week). Patients may fail 1 or 2 sessions of tDCS and/or CBT; and in that case, they will have the opportunity to receive the missing session (s) in the following week. The primary outcome will be assessed by week 2, using MADRS. Other assessments will be also performed by week 2, such as EEG, and other clinical measures (see Table 1). By week 4 and 6, patients will receive additional booster sessions of the same intervention (active or sham tDCS followed by CBT). Patients will perform the same assessments on week 6 (Figures 2A,B). tDCS sessions will follow the same design previously tested by two members of our group, that showed significant clinical effects of tDCS alone, and tDCS combined with sertraline in moderate to severe MDD (21).

Structured CBT sessions will follow the NICE guidelines from the National Institute for Health and Care Excellence (39). Patients will receive a total of 12 CBT sessions administered biweekly for 6 weeks. Mood, will be evaluated at the end of each week, as to perform the dose calculation (i.e., number of sessions) required to induce a clinical significant effect of at least $50 \%$ decrease in the MADRS scores. 


\section{Alnterventions and main assessments}

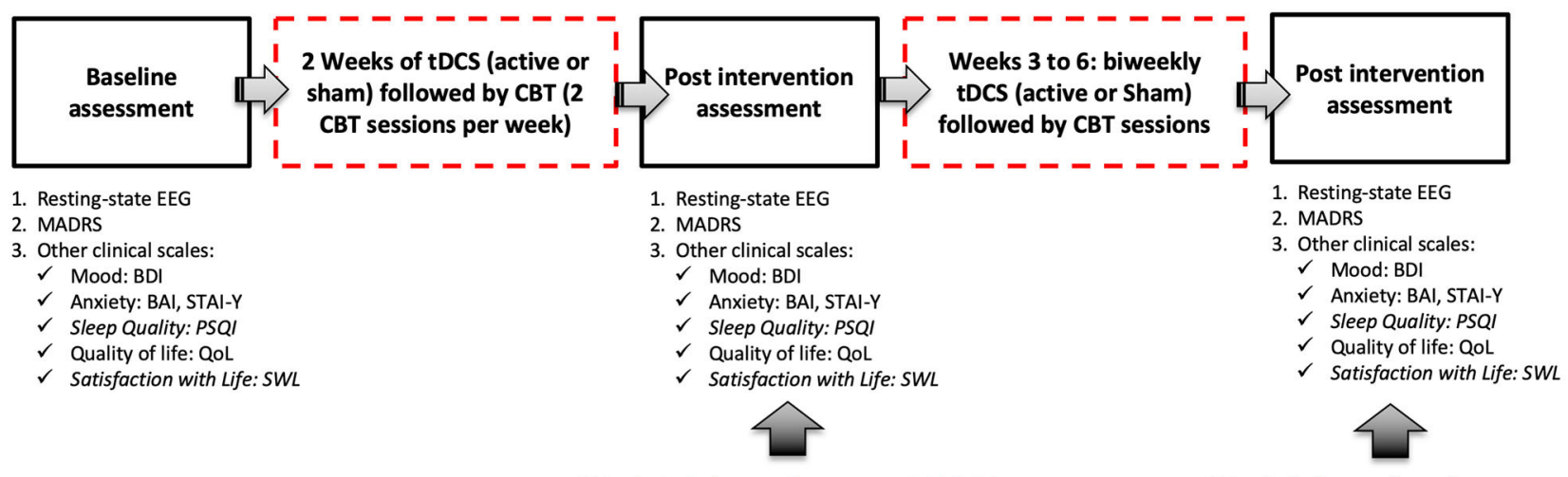

Week 2: Primary Outcome: MADRS

Week 6: Secondary Outcomes

\section{B Interventions}

Week 1 and Week 2
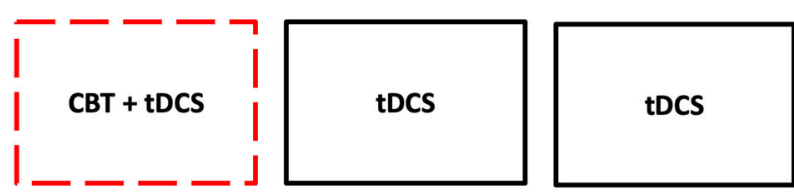

Total of 18 tDCs sessions

12 CBT sessions

\section{Week 3, 4. 5 and Week 6}
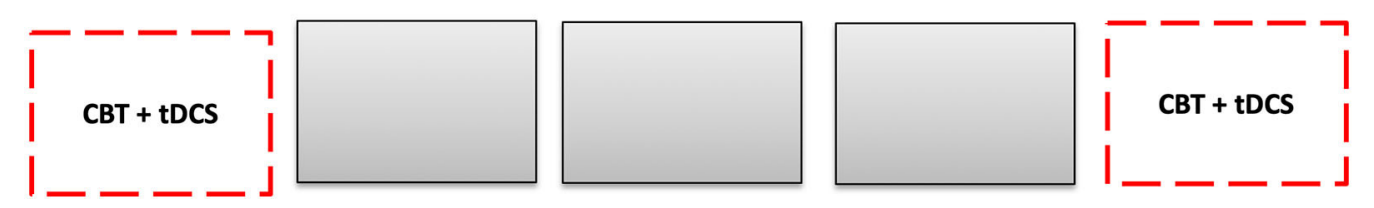

FIGURE 2 | Schematic representation of the main phases of the trial. (A) Shows the timeline of assessments. (B) Details how interventions sessions will be administered by week.

In addition to the primary outcome measure (MADRS), secondary outcome measures will be clinical response as measured by scores on the 17-item Hamilton Depression Rating Scale, Beck Depression Inventory (BDI), Beck Anxiety Inventory (BAI), clinician-rated Clinical Global Impression-Severity of Illness scale, and quality of life (QoL). These scales will be used at baseline, week 2, 4, 6, and follow-ups (up to 6-month follow up).

Resting-state EGG assessments will be performed on baseline session, on weeks 2 and 6 . These assessments will allow us to assess the neural effects of these interventions in the brain.

The Standard Protocol Items: recommendations for Interventional Trials (SPIRIT) statement were used as a framework for developing the study methodology for this trial $(40,41)$.

\section{Participants}

A total of 90 drug-naïve, first-episode MDD outpatients (45 per arm) will be randomized to receive either CBT combined with active bilateral tDCS over the DLPFC or CBT combined with sham tDCS.

\section{Eligibility Criteria}

Participants will be included in this study if they meet the following criteria: 1) Aged 18-75 years; 2) Unipolar, nonpsychotic MDD (DSM-V); 3) Score in the MADRS 7 and above (mild, moderate, and severe depression); 4) Low risk of suicide, as evaluated during the clinical interview and through the Scale for Suicidal Ideation [Mild to Moderate SIS; (42)]; and 5) Able to sign informed consent.

Potential participants meeting any of the following criteria will be excluded: 1) any contraindication to receive tDCS (such as metal in the head, implanted brain medical devices); 2) any significant or unstable neurologic or psychiatric disorder other than MDD, such as epilepsy, Parkinson's Disease, Dementias, eating disorders, OCD Spectrum disorders, among others); 3) history of substance abuse within the past 6-months, 4) Any personality disorders; or 5) any severe life-threatening disorders or concurrent medical condition likely to worsen 
patient's functional status in next 6-months such as; cancer, or severe heart, kidney, or liver diseases. Participants with reported high risk of suicide will be excluded from the study and will be recommended to receive support from an experience and licensed psychologist/psychiatrist. Information about local and national institutions that provide support to cope with suicidal behaviors and though will be provided.

A screening questionnaire that addresses the specific inclusion and exclusion criteria will be applied to each participant prior to the SCID-5 interview and will help to screen out participants. Diagnosis will be performed using the Structured Clinical Interview for DSM-5 (SCID-5) (43, 44), a widely used semi structured clinical interview designed to evaluate psychopathology, following the categories in the DSM 5 (Diagnostic and Statistical Manual of Mental Disorders, Fifth Edition) for the clinical use and clinical research. Potential participants will also answer the tDCS eligibility questionnaire and the SIS to evaluate the likelihood of suicide attempt. Patients with high likelihood of suicide attempt will be not included in our study and will be recommend for psychological and/or psychiatric intervention.

\section{Description of the Interventions Cognitive-Behavioral Therapy (CBT)}

CBT will be performed following the structure proposed by Beck et al. $(17,45)$. Each session will last $60 \mathrm{~min}$ and will be designed individually for each patient, according to the severity of Depression-including behavioral activation and problemsolving techniques. A licensed and trained practitioner will be responsible for facilitating the self-help programme and for reviewing progresses and outcomes during psychotherapy sessions. A senior clinical psychologist with extensive experience in CBT in patients with depression will supervise the clinical work. A clinical meeting will be hosted weekly in order to perform quality assurance of the therapeutic process.

\section{Transcranial Direct Current Stimulation (tDCS)}

tDCS will be delivered by an Eldith DC Stimulator Plus (Neuroconn, Germany), using $25 \mathrm{~cm}^{2}$ saline-soaked electrode sponges. Anode will be placed over the left (F3) and cathode over the right DLPFC (F4). For the active tDCS, participants will receive $2 \mathrm{~mA}$ (current density $=0.80 \mathrm{~A} / \mathrm{m}^{2}$; with $15 / 15 \mathrm{~s}$ $\mathrm{ramp} / \mathrm{ramp}$ down) for $30 \mathrm{~min} / \mathrm{d}$. For sham tDCS, patients will receive $15 \mathrm{~s}$ of $2 \mathrm{~mA}$ intensity, and $15 / 15 \mathrm{~s}$ ramp in and ramp down, with the same montage of electrodes; however, the device will be turned off after $45 \mathrm{~s}$ of active stimulation. Each tDCS session will last about $40 \mathrm{~min}$ : $30 \mathrm{~min}$ of stimulation and 10-min of set up. tDCS sessions will be performed by a research assistant, not involved in the CBT sessions.

\section{Description of the Assessments}

These instruments will be used at baseline, after the first 2 weeks of intervention, at the end of the 5-weeks of biweekly sessions and follow-up visits (up to 2 months after the intervention period) as detailed in the table below. Please see Table 1 for the complete assessments timetable.

\section{Outcomes}

\section{Primary Outcome}

For the depression assessment, we will use the MontgomeryAsberg Depression Scale (MADRS) as primary outcomes. Criteria for the use of this clinical scale will be the same as we previously used in Brunoni et al. (21). Secondary outcomes will include also the clinical response (categorical variable, defined as $>50 \%$ reduction of the baseline MADRS score), clinical remission (categorical variable, defined as a MADRS scores $\leq 10$ ), and scores on the BDI.

\section{Secondary Outcomes}

Resting state EEG and task-elicited prefrontal EEG alpha asymmetry-Resting state EEG screening will be carried out before the intervention (baseline), and in the end of each week of intervention. Each screening will comprise a resting state EEG ( 3 min eyes open and 3 min eyes closed) and a task-related EEG data collection. The task-related screening will last about 3-min in an open-eyes active state. For this task, we will use a facial emotion task, with approach and avoidance facial expressions, similar to the task used by Stewart and colleagues

EEG will be acquired using 20 channel Starstim (Neuroelectrics, Barcelona, Spain), following the 10/20 system, in a continuous mode at a digitization rate of $500 \mathrm{~Hz}$, with a bandpass filter of $0.01-100 \mathrm{~Hz}$. Electrode impedances will be kept below $5 \mathrm{k} \Omega$ and EOG will be recorded from two additional bipolar channels. EEG data will be segmented into 1.5-3 s epochs centered on subject's responses (at least 50 epochs) using EEGlab.

\section{Sample Size Calculation}

For the sample size calculation, we assume an effect size(d) of 0.66 [upper limit for the $95 \%$ CI of the pooled effect size of tDCS on MDD (22) that for a two-side $\alpha$ of 0.05 and a power of $80 \%$, requires a total of 76 patients (38 per group). We increased the sample size by $15 \%$ to 45 per group to account for unexpected factors. This sample size will be adequate to detect this magnitude of effect.

\section{Recruitment}

The enrollment of patients will be performed mainly from our Clinical Service at the School of Psychology, and by referral from Primary Care Physicians. After initial referral, potential patients with first episode MDD as primary diagnosis, will undergo the general inclusion and exclusion criteria check list in order to assess their potential eligibility.

We will also use social media (such as the Lab Facebook page), as well as flyers posted in specific spaces such as clinical settings (hospital, clinics), Universities, etc. The first screening will be performed by a research assistant (with a clinical Psychology degree) and the full assessment of the patient will be done by a clinician specialized in MDD (blinded to the study arm).

\section{Randomization}

Once eligibility and consent have been approved and obtained, randomization will occur using the randomized list generated by an automatic web-based randomization program. Patients 
TABLE 1 | Summary and timeline of assessments.

\begin{tabular}{|c|c|c|c|c|c|c|c|c|c|c|}
\hline & $\begin{array}{l}\text { Consent and } \\
\text { Screening }\end{array}$ & $\begin{array}{l}\text { Assessment } \\
\text { pre-intervention }\end{array}$ & $\begin{array}{l}\text { EEG pre- } \\
\text { intervention }\end{array}$ & $\begin{array}{l}\text { Interventions } \\
\text { Session and tDCS }\end{array}$ & $\begin{array}{l}\text { Assessment } \\
15 \text { days }\end{array}$ & $\begin{array}{l}\text { EEG } 15 \\
\text { days }\end{array}$ & $\begin{array}{l}\text { Interventions } \\
\text { Session and } \\
\text { tDCS }\end{array}$ & $\begin{array}{l}\text { Assessment } \\
\text { post- } \\
\text { intervention }\end{array}$ & $\begin{array}{l}\text { EEG post- } \\
\text { intervention }\end{array}$ & Follow-ups \\
\hline & & & & Daily Sessions & & & $\begin{array}{l}\text { Bi-weekly } \\
\text { sessions }\end{array}$ & & & $\begin{array}{l}15 \text { day, 1, 2, 3, } \\
\text { and } 5 \text { months }\end{array}$ \\
\hline & Email / Visit 1 & Visit 1 & Visit 2 & Visit 3-12 & Visit 1 & & Visit 14-21 & \multicolumn{2}{|c|}{ Visit 22} & Visit $24-27$ \\
\hline Approximate visit time & $1 \mathrm{~h} 30 \mathrm{~min}$ & $30 \mathrm{~min}$ & $1 h$ & $50 \mathrm{~min}$ & $30 \mathrm{mi}$ & & $50 \mathrm{~min}$ & \multicolumn{2}{|c|}{$30 \mathrm{~min}$} & $30 \mathrm{~min}$ \\
\hline
\end{tabular}

\section{Pre-screening questionnaires $X$}

(inclusion and exclusion

criteria)

Consent Informed (2 copies) X

Questionnaire eligibility for the $\mathrm{X}$

study

Questionnaire eligibility to tDCS $\mathrm{x}$

Questionnaire eligibility to EEG $\quad X$

Demographics and medical

questionnaire

The structured clinical interview

for DSM-5 (SCID-V)

PRIMARY AND SECONDARY OUTCOME ASSESSMENTS

The satisfaction with life scale

(SWL)

The pittsburgh sleep quality

index (PSQl)

State-trait anxiety inventory

(STAI-Y)

Beck anxiety inventory (BAl)

Beck's depression inventory

(BDI)

Montgomery-asberg

depression rating scale

(MADRS)

Blinding questionnaire

Visual analog scales

(VAS) - pre and post

$X$

$x$ $x$ $x$

$x$

$x$

$x$

$x$

X $x$ 
will be randomly assigned to one of the two groups in a 1:1 allocation ratio. Randomization order will be kept in sealed envelopes; therefore, patients will get their assignment according to the order of entrance in the study. This will also ensure that all patients and all other investigators are kept blind to this assignment for the duration of the study (allocation concealment) (see Figure 1, for study overview).

The randomization procedures described above will be followed for assignment to treatment groups. Following initial screening, during participant enrollment, a research associate will assign them to their randomly generated treatment group, keeping all patients and all other investigators blind to this assignment for the duration of the study.

\section{Blinding Procedure and Assessment}

Participants, the psychologists performing the CBT, the ones performing the assessments, as well the statistician will remain blinded to the tDCS condition up to the end of the clinical trial, ensuring a triple blind design. Researchers applying tDCS will not be blinded. If a serious adverse event occurs, the Principal Investigator (PI) will be responsible for removing the blinding and notify the Ethics Committee within $24 \mathrm{~h}$.

Blinding assessment will be performed to both participants and researchers who assessed the outcomes.

\section{Assessments}

\section{Eligibility and tDCS Assessments}

Questionnaire to assess eligibility to participate in the study: this questionnaire aims to evaluate inclusion and exclusion criteria to participate in the study. It includes specific questions about neurologic and psychiatric history, history of head injuries, drugs use, and/or abuse, history of treatments, etc.

Side Effects Questionnaire: At each stimulation session, patients will complete a questionnaire to evaluate potential adverse effects of tDCS (tingling, burning sensation, headache, neck pain, mood alterations). If any side effects are reported, the degree of relatedness to the intervention will be assessed on a 5-point scale. This type of adverse events questionnaire has been used frequently in our previous tDCS studies (46-52) including in patients with MDD. In order to further control for changes in suicidal thoughts, we will add a specific question for suicide that can be follow-up with the SSI, if scores are equal to or $>3$.

The Structured Clinical Interview for DSM 5 (SCID 5): this is a semi-structured clinical interview (the clinician version) that guides the clinician step-by-step through the DSM-5 diagnosis process. This interview will be essential to confirm the diagnosis of first episode MDD and to evaluate for possible comorbid psychiatric disorders (43).

Scale for suicide ideation (SSI): this is a 19-item scale that aims to quantify and assess suicidal intention (42). Patients scoring SSI $\geq 6$ will not be enrolled in the study (53).

$t D C S$ blinding questionnaire: After the treatment has ended, patients will complete a questionnaire to determine if our blinding methods were effective. We are using a $30 \mathrm{~s}$ sham montage, just as we use in our other trials, keeping the device on the subject for the duration of the session. The tDCS blinding questionnaire is organized in two main questions: 1. Please answer the questions to the best of your ability: 1.1 Did you receive: Sham Stimulation (tDCS) or Active Stimulation (tDCS); 1.2 Please, rate how confident you feel in your answer (please check one), from 1 (not confident at all), 2, 3 (somewhat confident), 4, to 5 (completely confident).

\section{Demographic and Clinical Assessments}

Demographics information: We will record information about the demographic characteristics of the study population such as age, gender, race, level of education, and social status.

Medication Use Log: Medication use information will be obtained at enrollment and updated on a weekly basis, by means of a Medication Log. Participants will record their current medications and dosages weekly, until completion of the study. Medication diaries are commonly used to record changes in medication use during the study period. We will also use the Antidepressant History Treatment Form (ATHF) to assess treatment refractoriness.

Montgomery-Asberg Depression Scale (MADRS): For the depression improvement assessments, we will use the MADRS for the primary outcome. This is widely used scale for the measurement of severity of depressive symptoms in patients with MDD. The scale is divided into 10 items, each scored on a 0 to 6-point ordinal scale (54). The MADRS will be administered according to a structured interview procedure that has been empirically found to result in high inter-rater reliability scores.

Beck Depression Inventory (BDI): This self-report inventory consists of 21 multiple-choice questions and is a widely used method to classify depression severity (55). It assesses for the presence of several symptoms related to depression, such as irritability, hopelessness and decreased cognitive performance. Physical symptoms such as weight loss and fatigue are also included. The total time required to complete this inventory is 5 to $10 \mathrm{~min}$.

Quality of Life Assessment (Short version of SF-36): The short version of the SF-36 health survey is used as a measurement of quality of life. It provides a profile of functional health and wellbeing scores. It is also used as a psychometrical index of physical and mental health (56).

Satisfaction with Life (SWL) - is a short and rapid 7-point Likert scale that measures life satisfaction in the perspective of subjective well-being. Scores in SWL have been positively correlated with measures of mental health and also predictive of future maladaptive behaviors such as suicide attempts $(57,58)$.

The Pittsburgh Sleep Quality Index (PSQI): a 19-item, selfreport measure to evaluate overall sleep quality, primarily designed to evaluate sleep disturbance in patients with psychiatric disorders. The PSQI evaluates sleep quality in 7 categories: subjective sleep quality, sleep latency, sleep duration, habitual sleep efficiency, sleep disturbances, use of sleep medication, and daytime sleep dysfunction $(59,60)$.

State-Trait Anxiety Inventory (STAI-Y) - a self-report measure is organized in two subscales: State Anxiety Scale (S-Anxiety) wish measures the current ("right now") presence and severity (state) of anxiety; and the Trait Anxiety Scale (T-Anxiety) which measures the general propensity to be anxious (trait). This 
measures focus on several domains that characterizes anxiety, such as subjective feelings, and levels of arousal (activations of the autonomic nervous systems). The trait subscale evaluates the more stable aspects of anxiety ("anxiety proneness"), such for instance worry, confidence and security (61).

Beck Anxiety Inventory (BAI): is a 21-item measure of anxiety focusing on somatic symptoms of anxiety. Each item is descriptive of somatic, subjective or panic-related symptoms of anxiety. Administration of BAI takes between 5 to $10 \mathrm{~min}$. Since BAI focus on a "pure" measure of anxiety such as nervousness, dizziness, inability to relax, etc.), it helps discriminating between depression and anxiety $(62,63)$.

\section{Data Management and Access to Data}

Data forms and questionnaires will be coded in a standardized manner, and double-entered in a protected excel sheet. Personal information and all data collect will be kept in locked cabinets that only the principal investigator will have access. A key to access these cabinets will be kept in a safe place with limited access. Only researchers involved in the study and any public health and safety authorities will have access to the data collected in the study. Any information linking data back to the participant will be discarded to ensure that the data are truly anonymous. Data destruction will be conducted 5 years after the study has ended. Data sharing will only be possible after an agreement. The data will be stored and managed following the GDPR in the EU. According to national regulations and the Ethics Committee approval, no Data Monitoring Committee (DMC) will accompany this study.

\section{Statistical Analysis \\ Primary Outcome}

A mixed model ANOVAs will be used to assess the clinical effects for primary outcome measure (MADRS), with intervention as between subject factor (active vs. sham tDCS), and time as within subject factor (weeks $2,4,8,12$, and 6 months). If there are significant baseline differences across the groups, those covariates will be included in the analysis (such as severity, anxiety, and quality of life). If significant main effects and/or interactions arise from the main analysis, post-hoc analysis corrected for multiple comparisons will be performed. If our hypothesis of prefrontal inter-hemispheric imbalance (as assessed by alpha power EEG) is confirmed, we will then conduct correlation analysis in order to assess whether these brain signatures correlate to long-term clinical effects in MDD.

\section{Secondary Outcomes \\ EEG Analysis}

To evaluate the degree of coupling between electrode pairs, we will use Magnitude Square Coherence as a pairwise connectivity measurement. For power spectrum, band power, and intraband mean and median analysis of the EEG frequency ranges, we will use the Fast Fourier transformation analysis, which will allow us to determine and measure the amplitude of the predominant EEG frequency, and properties in the time and frequency domains. For these two EEG analysis, we will define the following frequency bands: delta $(1-4 \mathrm{~Hz})$, theta $(4-8 \mathrm{~Hz})$, alpha $(8-13 \mathrm{~Hz})$, and beta $(13-30 \mathrm{~Hz})$ and four frequency sub-bands: low-alpha (9-10 Hz), high-alpha (10-12 Hz), low-beta $(13-20 \mathrm{~Hz})$, and highbeta $(20-30 \mathrm{~Hz})$, which can be obtained by decomposing the raw signal being generated in different areas of the brain.

Independent Component Analysis (ICA) will be used for artifact rejection. EEG changes during task will be assessed via task-related power (TRP)-i.e., TRP at a given electrode will be obtained by subtracting (log-transformed) power during a pre-stimulus reference interval from (log-transformed) power during the task. Power estimates will be obtained by squaring filtered EEG signals and then band power values will be averaged for both the pre-stimulus reference period and the task intervals. Degree of coupling between electrode pairs, will be assessed by using Magnitude Square Coherence as a pair-wise connectivity measurement.

\section{Ethics and Dissemination}

The trial is registered with the U.S. National Library of Medicine Clinical Trials Registry: NCT03548545 (ClinicalTrials.gov) and is approved by the local ethics committee-Subcomissão de Ética para as Ciências da Vida e da Saúde (SECVS) - SECVS 174/2017.

\section{Confidentiality}

All participants will be given subject identification codes composed by letters and numbers to which all the data will be linked. The file records that connect each participant to their identification number will be securely kept on University servers during the entire period of the study, and up to 5 years the study has ended.

\section{Dissemination Policy}

Results of this study will be published in peer reviewed journals, and will be disseminated in national and international conferences and in social media. In any of the dissemination procedures, subjects will not be identified or notified about the event.

\section{DISCUSSION}

The current study describes a protocol for a parallel randomized, triple-blind, sham controlled clinical trial to test the synergetic clinical and electrophysiology effects of combining cognitivebehavioral therapy with transcranial direct current stimulation in drug-naïve, first-episode MDD patients.

By combining two therapies that have shown promising results in patients with $\mathrm{MDD}$, we expect that the group that received CBT combined with active tDCS will have a greater reduction on MADRS scores, and will require lesser number of sessions in order for the clinical outcome to be reached. This result will have a significant impact since major depression is the second most prevalent mental disorder, which is thought to affect 163 million people worldwide (64). Furthermore, in Portugal, $7 \%$ of the population is diagnosed with depression every year (65), and suicide is responsible for more than a thousand deaths annually (66).

Despite the fact that tDCS has some promising effects on mood, it seems that it is in the combination of tDCS with other 
intervention that the effects are larger. For instance, in the study of Loo et al. (67), which randomized 64 patients to 15 sessions of $2 \mathrm{~mA}$ tDCS over 3 weeks and the study of Brunoni et al. (21), which enrolled 120 antidepressant-free patients with moderate and severe depression, tDCS has been shown to be effective in MDD. Moreover, in the study of Brunoni et al. (21), not only active tDCS was superior to sham tDCS but also the combined tDCS/sertraline was significantly more effective than in the other treatment groups in reducing depressive symptoms. Thus, the effects of tDCS seem to be enhanced by the combination with other interventions. This can be particularly important, if patient level data is taken into consideration. In a recent individual patient data meta-analysis (23) tDCS was shown to be less effective in high-resistant patients. This also reinforces the need of improving tDCS techniques so they can be effective in a broader depressed population.

We also propose to study, as secondary aim, whether CBT combined with tDCS can engage the proposed mechanistic target, of restoring the prefrontal imbalance and connectivity, by changes over resting-state and task-eliciting EEG. This trial will help to evaluate the efficacy of this combined treatment as compared to $\mathrm{CBT}$ alone and to evaluate bilateral alpha activity over the prefrontal cortex. Thus, we also expect to demonstrate that these interventions are able to reduce the interhemispheric asymmetry of alpha EEG activity toward the left hemisphere reported in patients with depression. We expect that the combined intervention will induce greater asymmetry reduction (at least 50\% minimum reduction). We also expect that this inter-hemispheric imbalance reduction will be correlated with mood improvement.

Furthermore, this mechanistic approach is one of the main advantages of the current proposal. For instance, the use of EEG as an adjuvant tool to exclude neurological conditions or to help in the diagnosis of psychiatric disorders is a common practice in the clinical setting. Evidence of abnormal findings are obtained in about 64 to $68 \%$ of the EEGs performed in psychiatric patients (68). These results suggest that EEG can be a potential technique to be used as a coadjutant for the diagnosis and prognosis of several psychiatric conditions, with potential reliability to guide neural-based interventions. EEG has been shown to differentiate patients with MDD and non-depressed healthy controls. Numerous studies have shown that depressed individuals present an asymmetry of EEG alpha activity toward the left hemisphere, even among previously depressed individuals when compared to those who have never experienced clinically significant depression (7-9). Some studies have nevertheless failed to show the link between decreased left frontal activation and depression (19). It has been suggested that inconsistencies in this literature may be the result of clinical and/or methodological differences between laboratories, such as the inclusion or exclusion of co-morbidities (such as anxiety disorders), gender related differences, or the choice of EEG reference $(9,69,70)$. Nonetheless, there is robust evidence suggesting that EEG alpha asymmetry is present among individuals with present or history of clinical depression, or even susceptibility to develop depression in the future (71). Additionally, alpha power asymmetry-based neurofeedback
(NFB), which aims to train patients to increase right-to-left ratio by rebalancing the left hemisphere hypoactivation, has shown promising results in $\operatorname{MDD}(72,73)$.

The advantages of EEG in MDD go far beyond its potential to detect differences between depressed vs. non-depressed individuals. Namely, EEG can be used to detect changes in the EEG patterns after interventions, and as such can be used to determine the efficacy of the intervention or if used also in the baseline, as an outcome predictor $(74,75)$. Furthermore, EEG is a direct measure of neural activity, it allows for chronometric sensitivity, has the potential to assess local and network effects, it is easy to use, cheap, and noninvasive. By using EEG, it is possible to quantify the electrical activity over specify regions of interest (power and coherence analysis) and, therefore, correlate with symptoms severity and response prognostic to specific treatments. Thus, creating brain based interventions.

These changes in EEG patterns have been used to direct several interventions, such as tDCS or TMS, however most study lack in the assessment of the real changes in EEG activity. For instance, based on this neurobiological basis, the main target for treating depressive symptoms using non-invasive brain stimulation (NIBS) techniques, such as tDCS and TMS, have been both the left (hypoactive) and right (hyperactive) DLPFC, by placing anodal (excitatory) over the left and cathodal (inhibitory) over the right DLPFC $(76,77)$. Using this mechanistic approach of facilitating the activation of the left DLPFC relative to the right, beneficial emotional, and cognitive effects in MDD were shown emotional and cognitive effects in $\operatorname{MDD}(18,78,79)$. Moreover, we chose to include adults with first episode of MDD only as to increase homogeneity of our study sample and thus increase internal validity of our findings. There are several advantages of studying the combination of these treatments in first episode MD drug-naive patients, as their brains were not changed by the pharmacological treatments, by the time course of the condition, or by the recurrence of MDD episodes (and other comorbidities). Second, combining tDCS and CBT are two therapies that have been shown to improve MDD. Moreover, tDCS is a safe, of easy administration, and not expensive noninvasive brain stimulation technique that has been shown to be effective in neuromodulating our target mechanism (imbalance over the DLPFC). Also, CBT is the golden standard treatment for MDD and has also shown to be able to neuromodulate brain structures involved in MDD. Thus, if we show that tDCS combined with CBT produce greater significant clinical improvements in MDD, this may reduce the global burden of MDD (for instance, by reducing the number of therapy sessions and the number of relapses and by producing larger long-term effects).

Therefore, the results of this project will further provide important insights into the mechanisms underlying MDD. In sum, we will be able to study the mechanistic reason underlying differences between the add-on treatment group vs. CBT combined with sham tDCS. We chose a population that is not very well studied, namely patients drug-naïve, first-episode MDD with mild to moderate symptoms, because several studies failed to show the link between decreased left frontal activation 
and depression. By using resting state EEG, it will be possible to simultaneously understand the neural effects of the intervention, as well as potential response predictors for future trials.

\section{TRIALS STATUS}

This clinical trial is currently on the recruitment phase.

\section{AUTHOR CONTRIBUTIONS}

SC and JL designed and developed the study protocol. ÓFG, AB, AF-G, and FF provided inputs to the design and development

\section{REFERENCES}

1. Murray CJL, Abraham J, Ali MK, Alvarado M, Atkinson C, Baddour LM, et al. The state of uS health, 1990-2010: burden of diseases, injuries, risk factors. JAMA - J Am Med Assoc. (2013) 310:591-608. doi: 10.1001/jama.2013.13805

2. Charlson F, van Ommeren M, Flaxman A, Cornett J, Whiteford H, Saxena S. New wHO prevalence estimates of mental disorders in conflict settings: a systematic review and meta-analysis. Lancet. (2019) 394:2408. doi: 10.1016/S0140-6736(19)30934-1

3. World Health Organization. Depression. (2020). Available online at: https:// www.who.int/news-room/fact-sheets/detail/depression [accessed May 17, 2020].

4. Zimmerman M, Walsh E, Friedman M, Boerescu DA, Attiullah N. Identifying remission from depression on 3 self-Report scales. J Clin Psychiatry. (2017) 78:177-83. doi: 10.4088/JCP.16m10641

5. Rush AJ, Trivedi MH, Wisniewski SR, Nierenberg AA, Stewart JW, Warden $\mathrm{D}$, et al. Acute and longer-term outcomes in depressed outpatients requiring one or several treatment steps: a STAR*D report. Am J Psychiatry. (2006) 163:1905-17. doi: 10.1176/ajp.2006.163.11.1905

6. Papakostas GI. Tolerability of modern antidepressants. J Clin Psychiatry. (2008) 69:8-13.

7. Nusslock R, Shackman AJ, Harmon-Jones E, Alloy LB, Coan JA, Abramson LY. Cognitive vulnerability and frontal brain asymmetry: common predictors of first prospective depressive episode. J Abnorm Psychol. (2011) 120:497503. doi: $10.1037 / \mathrm{a} 0022940$

8. Stewart JL, Coan JA, Towers DN, Allen JJB. Frontal eEG asymmetry during emotional challenge differentiates individuals with and without lifetime major depressive disorder. I Affect Disord. (2011) 129:16774. doi: 10.1016/j.jad.2010.08.029

9. Stewart JL, Coan JA, Towers DN, Allen JJB. Resting and task-elicited prefrontal EEG alpha asymmetry in depression: support for the capability model. Psychophysiology. (2014) 51:446-55. doi: 10.1111/psyp. 12191

10. Fonseka TM, MacQueen GM, Kennedy SH. Neuroimaging biomarkers as predictors of treatment outcome in major depressive disorder. J Affect Disord. (2018) 233:21-35. doi: 10.1016/j.jad.2017.10.049

11. Lai CH. Promising neuroimaging biomarkers in depression. Psychiatry Investig. (2019) 16:662-70. doi: 10.30773/pi.2019.07.25.2

12. Kaster TS, Daskalakis ZJ, Noda Y, Knyahnytska Y, Downar J, Rajji TK, et al. Efficacy, tolerability, and cognitive effects of deep transcranial magnetic stimulation for late-life depression: a prospective randomized controlled trial. Neuropsychopharmacology. (2018) 43:2231-8. doi: 10.1038/s41386-0180121-x

13. Alexander ML, Alagapan S, Lugo CE, Mellin JM, Lustenberger C, Rubinow DR, et al. Double-blind, randomized pilot clinical trial targeting alpha oscillations with transcranial alternating current stimulation (tACS) for the treatment of major depressive disorder (MDD). Transl Psychiatry. (2019) 9:439. doi: 10.1038/s41398-019-0439-0

14. Corlier J, Carpenter LL, Wilson AC, Tirrell E, Gobin AP, Kavanaugh $\mathrm{B}$, et al. The relationship between individual alpha peak frequency and clinical outcome with repetitive transcranial magnetic stimulation (rTMS) of the protocol and contributed equally to this work. All authors read and approved the final manuscript. All authors contributed to the article and approved the submitted version.

\section{FUNDING}

This work was partially supported by FEDER funds through the Programa Operacional Factores de CompetitividadeCOMPETE and by national funds through FCT_-Fundação para a Ciência e a Tecnologia through the calls IF/00091/2015 and PTDC/PSI-ESP/29701/2017. The sponsors had no role in the study design, implementation, data analysis or publication.

treatment of major depressive disorder (MDD). Brain Stimul. (2019) 12:15728. doi: 10.1016/j.brs.2019.07.018

15. Jog MV, Wang DJJ, Narr KL. A review of transcranial direct current stimulation (tDCS) for the individualized treatment of depressive symptoms. Pers Med Psychiatry. (2019) 8:17-22. doi: 10.1016/j.pmip.2019.03.001

16. Evans J, Heron J, Lewis G, Araya R, Wolke D. Negative self-schemas and the onset of depression in women: longitudinal study. Br J Psychiatry. (2005) 186:302-7. doi: 10.1192/bjp.186.4.302

17. Beck AT, Dozois DJA. Cognitive therapy: current status and future directions. Annu Rev Med. (2011) 62:397-409. doi: 10.1146/annurev-med-052209-100032

18. Wolkenstein L, Plewnia C. Amelioration of cognitive control in depression by transcranial direct current stimulation. Biol Psychiatry. (2013) 73:64651. doi: 10.1016/j.biopsych.2012.10.010

19. Baehr E, Rosenfeld JP, Baehr R. The clinical use of an alpha asymmetry protocol in the neurofeedback treatment of depression: two case studies. $J$ Neurother. (1997) 2:10-23. doi: 10.1300/J184v02n03_02

20. Loo CK, Alonzo A, Martin D, Mitchell PB, Galvez V, Sachdev P. Transcranial direct current stimulation for depression: 3Week, randomised, sham-controlled trial. Br J Psychiatry. (2012) 200:52-9. doi: 10.1192/bjp.bp.111.097634

21. Brunoni AR, Valiengo L, Baccaro A, Zanão TA, De Oliveira JF, Goulart A, et al. The sertraline vs electrical current therapy for treating depression clinical study. JAMA Psychiatry. (2013) 70:383-91. doi: 10.1001/2013.jamapsychiatry.32

22. Fregni F, El-Hagrassy MM, Pacheco-Barrios K, Carvalho S, Leite J, Simis $\mathrm{M}$, et al. Evidence-based guidelines secondary meta-analysis for the use of transcranial direct current stimulation (tDCS) in neurological psychiatric disorders. Int J Neuropsychopharmacol. (2020). doi: 10.1093/ijnp/pyaa051. [Epub ahead of print].

23. Moffa AH, Brunoni AR, Fregni F, Palm U, Padberg F, Blumberger DM, et al. Safety and acceptability of transcranial direct current stimulation for the acute treatment of major depressive episodes: analysis of individual patient data. $J$ Affect Disord. (2017) 221:1-5. doi: 10.1016/j.jad.2017.06.021

24. Brunoni AR, Boggio PS, De Raedt R, Bensenor IM, Lotufo PA, Namur V, et al. Cognitive control therapy and transcranial direct current stimulation for depression: a randomized, double-blinded, controlled trial. J Affect Disord. (2014) 162:43-9. doi: 10.1016/j.jad.2014.03.026

25. Segrave RA, Arnold S, Hoy K, Fitzgerald PB. Concurrent cognitive control training augments the antidepressant efficacy of tDCS: a pilot study. Brain Stimul. (2014) 7:325-31. doi: 10.1016/j.brs.2013.12.008

26. Farb N, Anderson A, Ravindran A, Hawley L, Irving J, Mancuso E, et al. Prevention of relapse/recurrence in major depressive disorder with either mindfulness-based cognitive therapy or cognitive therapy. J Consult Clin Psychol. (2018) 86:200-4. doi: 10.1037/ccp0000266

27. Zhang Z, Zhang L, Zhang G, Jin J, Zheng Z. The effect of cBT and its modifications for relapse prevention in major depressive disorder: a systematic review and meta-analysis. BMC Psychiatry. (2018) 18:5. doi: 10.1186/s12888-018-1610-5

28. Vitiello B. Combined cognitive-behavioural therapy and pharmacotherapy for adolescent depression: does it improve 
outcomes compared with monotherapy? CNS Drugs. (2009) 23:271-80. doi: 10.2165/00023210-200923040-00001

29. Peselow ED, Tobia G, Karamians R, Pizano D, IsHak WW. Prophylactic efficacy of fluoxetine, escitalopram, sertraline, paroxetine, and concomitant psychotherapy in major depressive disorder: outcome after long-term followup. Psychiatry Res. (2015) 225:680-6. doi: 10.1016/j.psychres.2014.11.022

30. Manber R, Buysse DJ, Edinger J, Krystal A, Luther JF, Wisniewski SR, et al. Efficacy of cognitive-behavioral therapy for insomnia combined with antidepressant pharmacotherapy in patients with comorbid depression and insomnia: a randomized controlled trial. J Clin Psychiatry. (2016) 2016:e1316e1323. doi: $10.4088 / J C P .15 \mathrm{~m} 10244$

31. DeRubeis RJ, Siegle GJ, Hollon SD. Cognitive therapy versus medication for depression: treatment outcomes and neural mechanisms. Nat Rev Neurosci. (2008) 9:788-96. doi: 10.1038/nrn2345

32. Driessen E, Hollon SD. Cognitive behavioral therapy for mood disorders: efficacy, moderators and mediators. Psychiatr Clin North Am. (2010) 33:53755. doi: 10.1016/j.psc.2010.04.005

33. Yang Z, Oathes D, Linn KA, Bruce SE, Satterthwaite TD, Cook PA, et al. Cognitive behavioral therapy is associated with enhanced cognitive control network activity in major depression and posttraumatic stress disorder. Biol Psychiatry Cogn Neurosci Neuroimaging. (2018) 3:3119. doi: 10.1016/j.bpsc.2017.12.006

34. Wagner G, Schultz CC, Koch K, Schachtzabel C, Sauer H, Schlösser RG. Prefrontal cortical thickness in depressed patients with high-risk for suicidal behavior. J Psychiatr Res. (2012) 46:1449-55. doi: 10.1016/j.jpsychires.2012.07.013

35. Goldstein-Piekarski AN, Staveland BR, Ball TM, Yesavage J, Korgaonkar MS, Williams LM. Intrinsic functional connectivity predicts remission on antidepressants: a randomized controlled trial to identify clinically applicable imaging biomarkers. Transl Psychiatry. (2018) 8. doi: 10.1038/s41398-018-0100-3

36. Korgaonkar MS, Goldstein-Piekarski AN, Fornito A, Williams LM. Intrinsic connectomes are a predictive biomarker of remission in major depressive disorder. Mol Psychiatry. (2019) 25:1537-49. doi: 10.1038/s41380-019-0574-2

37. Jos,é C, Fernando B, Iván C. TMS for major depressive disorder in naïve patients. Brain Stimul. (2019) 12:e133. doi: 10.1016/j.brs.2019.03.037

38. Maneeton B, Maneeton N, Woottiluk P, Likhitsathian S. Repetitive transcranial magnetic stimulation combined with antidepressants for first episode of major depressive disorder. Curr Neuropharmacol. (2020) 2020:18. doi: 10.2174/1570159x18666200221113134

39. National Institute for Health Care Excellence. Depression in Adults: Recognition Management. NICE (2009). Available online at: https://www. nice.org.uk/guidance/cg90/chapter/1-Guidance\#treatment-choice-basedon-depression-subtypes-and-personal-characteristics [accessed August 25, 2020].

40. Chan AW, Tetzlaff JM, Gøtzsche PC, Altman DG, Mann H, Berlin JA, et al. SPIRIT 2013 explanation and elaboration: guidance for protocols of clinical trials. BMJ. (2013) 346:e7586. doi: 10.1136/bmj.e7586

41. Chan, A.-W., Tetzlaff JM, Altman DG, Laupacis A, Gøtzsche PC, et al. SPIRIT 2013 statement: defining standard protocol items for clinical trials. Ann Intern Med. (2013) 158:200. doi: 10.7326/0003-4819-158-3-201302050-00583

42. Beck AT, Kovacs M, Weissman A. Assessment of suicidal intention: the scale for suicide ideation. J Consult Clin Psychol. (1979) 47:34352. doi: 10.1037/0022-006X.47.2.343

43. Shankman SA, Funkhouser CJ, Klein DN, Davila J, Lerner D, Hee D. Reliability and validity of severity dimensions of psychopathology assessed using the Structured Clinical Interview for DSM-5 (SCID). Int J Methods Psychiatr Res. (2018) 2018:27. doi: 10.1002/mpr.1590

44. Osório FL, Loureiro SR, Hallak JEC, Machado-de-Sousa JP, Ushirohira JM, Baes CVW, et al. Clinical validity and intrarater and test-retest reliability of the structured clinical interview for dSM-5 - clinician version (SCID-5-CV). Psychiatry Clin Neurosci. (2019) 73:754-760. doi: 10.1111/pcn.12931

45. Beck AT, John Rush A, Shaw BF, Emery G. Cognitive Therapy of Depression, the Guilford Clinical Psychology and Psychopathology Series by Aaron. New York, NY: Booktopia. (1987). Available online at: https://www.guilford. com/books/Cognitive-Therapy-of-Depression/Beck-Rush-Shaw-Emery/ 9780898629194 (accessed May 13, 2020).
46. Leite J, Carvalho S, Fregni F, Boggio PS, Gonçalves ÓF. The effects of cross-hemispheric dorsolateral prefrontal cortex transcranial direct current stimulation (tDCS) on task switching. Brain Stimul. (2013) 2013:6. doi: 10.1016/j.brs.2012.10.006

47. Carvalho S, Gonçalves OF, Soares JM, Sampaio A, Macedo F, Fregni F, et al. Sustained effects of a neural-based intervention in a refractory case of tourette syndrome. Brain Stimul. (2015) 2015:8. doi: 10.1016/j.brs.2014.12.008

48. Carvalho S, Boggio PS, Gonçalves ÓF, Vigário AR, Faria M, Silva S, et al. Transcranial direct current stimulation based metaplasticity protocols in working memory. Brain Stimul. (2015) 2015:11. doi: 10.1016/j.brs.2014.11.011

49. Carvalho S, French M, Thibaut A, Lima W, Simis M, Leite J, et al. Median nerve stimulation induced motor learning in healthy adults: a study of timing of stimulation and type of learning. Eur J Neurosci. (2018) 48:13990. doi: 10.1111/ejn.13990

50. Carvalho S, Leite J, Jones F, Morse LR, Zafonte R, Fregni F. Study adherence in a tDCS longitudinal clinical trial with people with spinal cord injury. Spinal Cord. (2018) 56:5. doi: 10.1038/s41393-017-0023-5

51. Carvalho S, Sampaio A, Mendes AJ, Lema A, Vieira D, Goncalves OF, et al. Polarity specific effects of cross-hemispheric tDCS coupled with approach-avoidance training on chocolate craving. Front Pharmacol. (2019) 9:1500. doi: 10.3389/fphar.2018.01500

52. Thibaut A, Carvalho S, Morse LR, Zafonte R, Fregni F. Delayed pain decrease following $\mathrm{m} 1 \mathrm{tDCS}$ in spinal cord injury: a randomized controlled clinical trial. Neurosci Lett. (2017) 658:24. doi: 10.1016/j.neulet.2017.08.024

53. De Beurs DP, Fokkema M, O'Connor RC. Optimizing the assessment of suicidal behavior: the application of curtailment techniques. J Affect Disord. (2016) 196:218-24. doi: 10.1016/j.jad.2016.02.033

54. Montgomery SA, Asberg M. A new depression scale designed to be sensitive to change. Br J Psychiatry. (1979) 134:382-9. doi: 10.1192/bjp.134.4.382

55. Beck AT, Ward $\mathrm{CH}$, Mendelson M, Mock J, Erbaugh J. An inventory for measuring depression. Arch Gen Psychiatry. (1961) 4:561-71. doi: 10.1001/archpsyc.1961.01710120031004

56. Jenkinson C, Layte R, Jenkinson D, Lawrence K, Petersen S, Paice C, et al. A shorter form health survey: can the sf- 12 replicate results from the sf-36 in longitudinal studies? J Public Heal. (1997) 19:179-86. doi: 10.1093/oxfordjournals.pubmed.a024606

57. Diener E, Emmons RA, Larsem RJ, Griffin S. The satisfaction with life scale. J Pers Assess. (1985) 49:71-5. doi: 10.1207/s15327752jpa4901_13

58. Pavot W, Diener E, Colvin CR, Sandvik E. Further validation of the satisfaction with life scale; evidence for the cross-Method convergence of well-Being measures. J Pers Assess. (1991) 57:149-61. doi: 10.1207/s15327752jpa5701_17

59. Buysse DJ, Reynolds CF, Monk TH, Berman SR, Kupfer DJ. The pittsburgh sleep quality index: a new instrument for psychiatric practice and research. Psychiatry Res. (1989) 28:193-213. doi: 10.1016/0165-1781(89)90047-4

60. Del Rio João KA, Becker NB, de Neves Jesus S, Isabel Santos Martins R. Validation of the portuguese version of the pittsburgh sleep quality index (PSQI-PT). Psychiatry Res. (2017) 247:2259. doi: 10.1016/j.psychres.2016.11.042

61. Spielberger CD, Gorsuch RL, Lushene RE. Manual for the State-Trait Anxiety Inventory. Palo Alto, CA: Consulting Psychologists Press (1970).

62. Beck AT, Epstein N, Brown G, Steer RA. An inventory for measuring clinical anxiety: psychometric properties. J Consult Clin Psychol. (1988) 56:8937. doi: $10.1037 / 0022-006 X .56 .6 .893$

63. Quintão S, Delgado AR, Prieto G. Validity study of the beck anxiety inventory (Portuguese version) by the rasch rating scale model. Psicol Reflex e Crit. (2013) 26:305-10. doi: 10.1590/S0102-79722013000200010

64. James SL, Abate D, Abate KH, Abay SM, Abbafati C, Abbasi N, et al. Global, regional, national incidence. Prevalence, and years lived with disability for 354 Diseases and Injuries for 195 countries and territories, 1990-2017: A systematic analysis for the Global Burden of Disease Study 2017. Lancet. (2018) 392:1789-858. doi: 10.1016/S0140-6736(18)32279-7

65. de Almeida JC. Portuguese national mental health plan (2007-2016) executive summary. Ment Health Fam Med. (2009) 6:233-44.

66. Alonso J, Angermeyer MC, Bernert S, Bruffaerts R, Brugha TS, Bryson $\mathrm{H}$, et al. Disability and quality of life impact of mental disorders in europe: results from the european study of the epidemiology of mental 
disorders (ESEMeD) project. Acta Psychiatr Scand Suppl. (2004) 109:3846. doi: 10.1111/j.1600-0047.2004.00329.x

67. Loo CK, Husain MM, McDonald WM, Aaronson S, O'Reardon JP, Alonzo A, et al. International randomized-controlled trial of transcranial direct current stimulation in depression. Brain Stimul. (2018) 11:12533. doi: 10.1016/j.brs.2017.10.011

68. Newson JJ, Thiagarajan TC. EEG frequency bands in psychiatric disorders: a Review of resting state studies. Front Hum Neurosci. (2019) 12:521. doi: 10.3389/fnhum.2018.00521

69. Hagemann D. Individual differences in anterior eEG asymmetry: methodological problems and solutions. Biol Psychol. (2004) 67:157-82. doi: 10.1016/j.biopsycho.2004.03.006

70. Stewart JL, Bismark AW, Towers DN, Coan JA, Allen JJB. Resting frontal eEG asymmetry as an endophenotype for depression risk: sex-specific patterns of frontal brain asymmetry. J Abnorm Psychol. (2010) 119:50212. doi: 10.1037/a0019196

71. Allen JJB, Coan JA, Nazarian M. Issues and assumptions on the road from raw signals to metrics of frontal EEG asymmetry in emotion. Biol Psychol. (2004) 67:183-18. doi: 10.1016/j.biopsycho.2004.03.007

72. Rosenfeld JP, Cha G, Blair T, Gotlib IH. Operant (biofeedback) control of left-right frontal alpha power differences: potential neurotherapy for affective disorders. Biofeedback Self Regul. (1995) 20:241-58. doi: 10.1007/BF014 74516

73. Allen JJB, Harmon-Jones E, Cavender JH. Manipulation of frontal eEG asymmetry through biofeedback alters self-reported emotional responses and facial eMG. Psychophysiology. (2001) 38:685-93. doi: 10.1111/1469-8986.3840685

74. Hughes JR, John ER. Conventional and quantitative electroencephalography in psychiatry. J Neuropsychiatry Clin Neurosci. (1999) 11:190208. doi: 10.1176/jnp.11.2.190
75. Badrakalimuthu VR, Swamiraju R, De Waal H. EEG in psychiatric practice: to do or not to do? Adv Psychiatr Treat. (2011) 17:11421. doi: 10.1192/apt.bp.109.006916

76. Berlim MT, Van den Eynde F, Daskalakis ZJ. Clinical utility of transcranial direct current stimulation (tDCS) for treating major depression: a systematic review and meta-analysis of randomized, double-blind and sham-controlled trials. J Psychiatr Res. (2013) 47:1-7. doi: 10.1016/j.jpsychires.2012.09.025

77. Shiozawa P, Fregni F, Benseñor IM, Lotufo PA, Berlim MT, Daskalakis JZ, et al. Transcranial direct current stimulation for major depression: an updated systematic review and meta-analysis. Int J Neuropsychopharmacol. (2014) 17:1443-52. doi: 10.1017/S1461145714000418

78. Terhune DB, Cohen Kadosh R. Modulating cognitive control in major depression with transcranial electrical stimulation. Biol Psychiatry. (2013) 73:595-6. doi: 10.1016/j.biopsych.2013.01.006

79. Moreno ML, Vanderhasselt MA, Carvalho AF, Moffa AH, Lotufo PA, Benseñor IM, et al. Effects of acute transcranial direct current stimulation in hot and cold working memory tasks in healthy and depressed subjects. Neurosci Lett. (2015) 591:126-31. doi: 10.1016/j.neulet.2015.02.036

Conflict of Interest: The authors declare that the research was conducted in the absence of any commercial or financial relationships that could be construed as a potential conflict of interest.

Copyright (c) 2020 Carvalho, Gonçalves, Brunoni, Fernandes-Gonçalves, Fregni and Leite. This is an open-access article distributed under the terms of the Creative Commons Attribution License (CC BY). The use, distribution or reproduction in other forums is permitted, provided the original author(s) and the copyright owner(s) are credited and that the original publication in this journal is cited, in accordance with accepted academic practice. No use, distribution or reproduction is permitted which does not comply with these terms. 